\section{Weniger Darmkrebstote durch Polypektomie?}

Was bringt die direkte Polypenentfernung bei der Dickdarmspiegelung für die durch Kolorektalkarzinome bedingte Mortalität? Dieser Frage ging man in der US-amerikanischen „National Polyp Study“ nach.

ei 2.602 Patienten, die aus verschiedenen Verdachtsgründen zu einer ersten Koloskopie überwiesen worden waren, wurden zwischen 1980 und 1990 an sieben Kliniken Adenome entfernt. $42,7 \%$ der Adenome waren klein, $57,3 \%$ befanden sich in einem fortgeschrittenen Stadium (z. B. Durchmesser $\geq 1 \mathrm{~cm}$ ). Meist wurde nur ein Adenom entdeckt

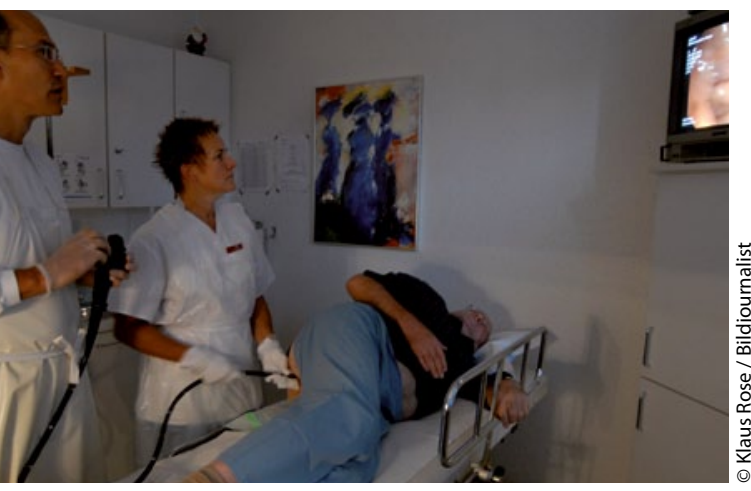

Die Entfernung von Adenomen kann das Risiko für ein Kolorektalkarzinom senken.
(58,6\%), bei $22,1 \%$ der Patienten zwei und bei 19,3\% drei oder mehr. Die Nachbeobachtungszeit lag im Median bei 15,8 und maximal bei 23 Jahren.

Nach knapp 16 Jahren waren 1.246 der Patienten (48\%) verstorben, zwölf an einem Kolorektalkarzinom. In der Allgemeinbevölkerung wäre im gleichen Zeitraum (nach den SEER-Daten) mit rund 25 Todesfällen infolge Darmkrebs zu rechnen gewesen. So verringerte sich die krankheitsspezifische Mortalitätsrate nach Polypektomie um $53 \%$. Die Sterberaten lagen zehn Jahre nach dem Eingriff zudem ähnlich hoch wie bei den $773 \mathrm{~Pa}$ tienten der internen Kontrollgruppe, bei denen nach Polypentfernung kein Adenom, sondern z. B. ein hyperplastischer Polyp diagnostiziert wurde. Die lange Nachbeobachtungszeit der Studie stütze die Hypothese, dass mit der kolonoskopischen Entfernung von Polypen die Mortalität des Kolorektalkarzinoms gesenkt werden könne, so die Autoren.
Adenome gelten als Risikofaktor für die Entwicklung des Kolorektalkarzinoms. Die Studiendaten hätten gezeigt, dass die Entfernung der Adenome und die anschließende Überwachung der Patienten dieses Risiko senken könne, meinten norwegische Autoren in einem begleitenden Editorial. Sie sehen es als reizvolles Konzept an, die Koloskopie allen 60-Jährigen einmal anzubieten. Würden keine Adenome diagnostiziert, müssten die Patienten nicht mehr untersucht werden. Würden dagegen Adenome festgestellt, würden die Betroffenen als Hochrisikopatienten eingestuft und nach Entfernung der Geschwülste weiterhin eng überwacht.

Fazit: Trotz des plausiblen Ergebnisses der Studie, so die Editorialisten, seien, um die Effektivität eines Screenings auf Populationsebene zu untersuchen, randomisierte, populationsbasierte Studien erforderlich.

Christine Starostzik

Zauber AG et al. Colonoscopic polypectomy and long-term prevention of colorectal-cancer deaths. N Engl J Med. 2012; 366(8): 68796. - Editorial: Bretthauer M, Kalager M. Colonoscopy as a triage screening test. Ibidem 759-60.

\title{
Bei Rektumkarzinom präoperativ Bevacizumab?
}

Bei Patienten mit lokal fortgeschrittenem oder tiefem Rektumkarzinom ist das Rezidivrisiko hoch. Präoperative Chemoradiotherapie soll ein maximales Downstaging erzielen. Lohnt sich zusätzlich die Gabe von Bevacizumab?

$\mathrm{n}$ einer Phase-II-Studie ging es um Sicherheit und Wirksamkeit einer präoperativen Chemoradiotherapie unter Einschluss von Bevacizumab, einem monoklonalen Antikörper gegen den vaskulären endothelialen Wachstumsfaktor. Eingeschlossen waren 42 Patienten mit lokal fortgeschrittenem oder tiefem Rektumkarzinom, darunter 18 Patienten (43\%) mit klinischen T4- und/oder N2Tumoren. Sie erhielten Capecitabin 825 $\mathrm{mg} / \mathrm{m}^{2}$ zweimal täglich an den Tagen 1-14 und 22-35, Oxaliplatin $50 \mathrm{mg} / \mathrm{m}^{2}$ an den Tagen 1, 8, 22 und 29, Bevacizumab $5 \mathrm{mg} /$ kg zwei Wochen vor Beginn der Bestrahlung sowie an den Tagen 1, 15 und 29; die Bestrahlung bestand aus 50,4 Gy in 28 Fraktionen inklusive Boost. Im Mittel wurde eine relative Dosisintensität von über $90 \%$ für alle Chemotherapeutika und von $97 \%$ für die Bestrahlung erreicht.

Sieben bis neun Wochen nach Chemoradiotherapie unterzogen sich 38 Patienten wie geplant einer totalen mesorektalen Exzision. Bei 35 (92,1\%) war eine R0-Resektion möglich, bei drei eine R1Resektion (7,9\%). Eine komplette Tumorregression (ypT0) fand sich bei neun Patienten (23,7\%), da aber zwei Lymphknotenmetastasen aufwiesen, zeigten insgesamt sieben $(18,4 \%)$ ein pathologisch komplettes Ansprechen (ypT0N0). Durch die zentrale Überprüfung der Befunde änderte sich das pathologische Staging in sechs Fällen (16\%). In der Phase vor der Operation wurden Grad-3/4-
Diarrhöen bei zehn Patienten (24\%) und Schmerzen bei vier Patienten (10\%) registriert, während postoperativ bei jeweils fünf Patienten Schmerz Grad 3/4, Fatigue und Infektion im Vordergrund standen.

Fazit: Die präoperative Gabe von Bevacizumab zusätzlich zu Oxaliplatin, Capecitabin und Bestrahlung beim Hochrisiko-Rektumkarzinom war zwar sicher und die Rate von kompletter Tumorregression mit 23,7\% recht vielversprechend, der vorab definierte Wirksamkeitsendpunkt ( $\geq 25 \%$ ) wurde aber verfehlt. Eine PhaseIII-Studie scheint daher nicht gerechtfertigt. Die Ergebnisse weiterer Studien zur neoadjuvanten Gabe von Bevacizumab bleiben abzuwarten.

Ulrike Wepner

Kennecke $\mathrm{H}$ et al. Pre-operative bevacizumab, capecitabine, oxaliplatin and radiation among patients with locally advanced or low rectal cancer: a phase II trial.

Eur J Cancer. 2012;48(1):37-45. 